\title{
COPING WITH CHRONIC DISEASE? CHRONIC DISEASE AND DISABILITY IN ELDERLY AMERICAN POPULATION 1982-1999
}

\author{
Gabriel Aranovich \\ Jay Bhattacharya \\ Alan M. Garber \\ Thomas E. MaCurdy \\ Working Paper 14811 \\ http://www.nber.org/papers/w14811 \\ NATIONAL BUREAU OF ECONOMIC RESEARCH \\ 1050 Massachusetts Avenue \\ Cambridge, MA 02138 \\ March 2009
}

We thank David Wise for his comments on an earlier draft of this paper. We thank the National Institute on Aging for funding this project. The views expressed herein are those of the author(s) and do not necessarily reflect the views of the National Bureau of Economic Research.

NBER working papers are circulated for discussion and comment purposes. They have not been peerreviewed or been subject to the review by the NBER Board of Directors that accompanies official NBER publications.

(C) 2009 by Gabriel Aranovich, Jay Bhattacharya, Alan M. Garber, and Thomas E. MaCurdy. All rights reserved. Short sections of text, not to exceed two paragraphs, may be quoted without explicit permission provided that full credit, including $(\odot$ notice, is given to the source. 
Coping with Chronic Disease? Chronic Disease and Disability in Elderly American Population 1982-1999

Gabriel Aranovich, Jay Bhattacharya, Alan M. Garber, and Thomas E. MaCurdy

NBER Working Paper No. 14811

March 2009

JEL No. I1,I18

\begin{abstract}
It is well known that disability rates among the American elderly have declined over the past decades. The cause of this decline is less well established. In this paper, we test one important possible explanation-that the decline in disability occurred because of chronic disease prevention efforts among the elderly. For this purpose we analyze data from the National Long Term Care Survey and from the National Health and Interview Survey. Our findings suggest that primary prevention, as reflected in decreased disease prevalence, was not responsible for advances made in elderly functioning between 1980 and 2000. We found a broad decline in less severe forms of disability that is unlikely to have resulted from improved disease management. Instead, these measured improvements in functioning may reflect environmental, technological, and/or socioeconomic changes. Improvements in the more severe forms of disability were modest and were restricted to those suffering from particular illnesses, which make improved and/or more aggressive management a plausible explanation and one that might increase costs should the trend persist.
\end{abstract}

Gabriel Aranovich

Department of Psychiatry, UCSF

401 Parnassus Avenue, Box 0984-ADM

San Francisco, CA 94143-0984

garanovich@gmail.com

Jay Bhattacharya

117 Encina Commons

Center for Primary Care

and Outcomes Research

Stanford University

Stanford, CA 94305-6019

and NBER

jay@stanford.edu

\author{
Alan M. Garber \\ PCOR/CHP \\ 117 Encina Commons \\ Stanford, CA 94305-6019 \\ and NBER \\ garber@stanford.edu \\ Thomas E. MaCurdy \\ Department of Economics \\ Stanford University \\ Stanford, CA 94305-6072 \\ and NBER \\ tmac@leland.stanford.edu
}




\subsection{Introduction}

It has now become a widely-accepted finding, confirmed by review articles in the most prominent medical journals (Freedman, Martin, and Shoeni 2002), that American elderly are less likely to be disabled than they were two decades ago. This fact represents a sharp reversal from the consensus among researchers in the 1970 s that disability rates among the elderly were rising. In this older research (for example, Gruenberg 1977), the impressive mortality declines of the $20^{\text {th }}$ century represented a "failure of success" since it appeared that extra life years were spent by the elderly in a disabled state. The evidence over the last few decades has proven to be more in line with the contrary view, first articulated by Fries (1980) and known as "compression of morbidity." Fries predicted that morbidity would decline at a faster rate than mortality and, as a result, U.S. elderly would experience a foreshortening of the time spent in a functionally impaired state.

One aspect of Fries' theory that has not been borne out by the evidence is a predicted decline in the prevalence of chronic disease among American elderly. The data show a mixed trend. Desai et al. (1999) report that the leading causes of death in the U.S. over-65 population are heart disease, cancers, and strokes, all three of which have indeed declined in prevalence and associated mortality over the last decade. In contrast, asthma, hip fractures, arthritis, diabetes, and obesity, have become more prevalent. Other studies (Crimmins et al. 2000, Freedman and Martin 2000) found increases in reported rates of nearly all of the chronic medical conditions.

It has become clear that the decline in elderly disability rates is mostly accounted for by a decrease in its less severe forms. This assertion has received support from 
studies that decomposed disability into so-called Instrumental Activities of Daily Living (IADLs) and Activities of Daily Living (ADLs). IADLs include everyday behaviors such as grocery shopping, managing money, and preparing meals and are considered a measure of moderate disability. The ADL measure, which encompasses more basic, mechanically-oriented activities, including dressing, eating, and bathing, is considered a gauge of more severe forms of functional impairment. Studies that employed this distinction found significant decreases in IADL disability (Spillman 2003, Freedman M S 2002, Working group 2002). The ADL measure has produced equivocal results. Some studies have shown modest decreases in ADL disability while others have shown increases (Manton vs. Crimmins). Analyses of the National Long Term Care Survey (NLTCS) dataset have shown a decline in the prevalence of "any ADL", with an increase in the mean number of ADLs (Spillman 2003). Further analysis has also clarified the chronicity of functional improvements with greater declines observed during the 1990s in published studies of the 1980s and 1990s (working group, freedman 2002).

One area that remains to be elucidated is the role that disease prevention and management have played in improvements in elderly functioning. In particular, it is important to understand whether gains in functional status can be attributed to disease prevention or whether diseases have become less disabling. Freedman and Martin (2000) examined various chronic disabling conditions using the National Health Interview Survey (NHIS) Supplement on Aging datasets from 1984 and 1994. They explored the impact of osteoporosis, broken hip, hypertension, stroke, cancer, diabetes, heart disease, arthritis, and obesity on functional status. Their findings included evidence of increases in prevalence among all conditions except hypertension. In addition, they found 
statistically significant declines in the debilitating effects of osteoporosis, heart disease, and arthritis. Cancer was found to be more disabling in the latter year. They propose that declines in the debilitating effects of chronic illnesses help to explain the broad reduction in elderly disability. Using the same dataset, Crimmins and Saito (2000) compared ADL, IADL, and Nagi measures of disability. Nagi functions measure self-reported difficulty with physical tasks such as walking up stairs and are believed to indicate less severe functioning loss. In contrast to Freedman and Martin, Crimmins and Saito found that arthritis had become more debilitating in the context of ADL impairments. The only significant changes in functioning among chronically ill men represented increased functional impairment. Elderly men with diabetes, arthritis, stroke, and cataracts had more ADL disability in 1994 than in 1984. Women with arthritis, osteoporosis, hypertension, and hearing loss also had more ADL disability on average in the latter year. However, women with heart disease, stroke, cancer, arthritis, osteoporosis, hypertension, and/or glaucoma all had significantly less IADL and Nagi disability in 1994 than in 1984.

In this study we update these papers with data from the early 1980s through 1999. We use data from both the NLTCS and NHIS to estimate trends in the likelihood of disability given chronic illness among U.S. elderly and decompose the changes into the IADL and ADL categories of disability. Our study focuses on the role of arthritis, diabetes, hypertension, heart disease, stroke, overweight, and chronic obstructive lung disease (COPD). We estimate prevalence of chronic illness, prevalence of disability, and prevalence of disability given chronic illness. Whereas Freedman et al. and Crimmins et al. used regression analysis, we calculate estimates without making such stringent assumptions about functional form. 


\subsection{Background}

There is broad literature looking at trends in disability in the American elderly population over the past two decades. Contributors to this literature have relied upon different surveys and different definitions of disability, but have consistently found declines, sometimes sharp and accelerating declines in disability among the elderly.

Manton, Corder, and Stallard (1997) use the 1982, '84, '89, and '94 National Long Term Care Surveys (NLTCS) to investigate trends in the prevalence of disability in the elderly population. Defining disability as an inability to perform an ADL/IADL without aid for at least 90 days, they find that the age-adjusted prevalence of disability for 1994 decreased by $3.6 \%$ from 1982 (from $24.9 \%$ to $21.3 \%$ ). The authors compare the size of the set of people with disabilities with that which would have occurred without the apparent declines in disability. There were 0.54 million and 1.2 million fewer disabled in 1989 and 1994, respectively, than there would have been had the 1982 rates stayed fixed (Manton et al., 1993 and 1997). Manton and Gu (2001) update these results using the latest wave of the NLTCS. They confirm a continuing decline in disability among the elderly, especially among the oldest age groups.

Manton et al. also investigate the incidence of disability and the progression to increased forms of disability. They calculate the rates of being institutionalized from having 1-2 ADLs, 3-4 ADLs, 5-6 ADLs, or some number of IADLs exclusively in 1982 and 1984. Decline in disability accelerated from $0.27 \%$ per year from 1982 to ' 89 to 0.34\% from 1989 to 1994, with greater decline in older cohorts (Manton and Gu, 2001). The transition rates from 1982-1984 and from 1984-'89 were also improved (Manton, Corder, Stallard 1993). Manton (2003) and Pardes et al. (1999) attribute these recent 
improvements in disability prevalence among the elderly to improvements in medical technology that enable seniors to delay both disability and death. They argues strongly that potential future medical breakthroughs (such as the treatment and prevention of senile dementia) hold the promise of further disability reductions for the elderly.

Freedman and Martin (1998) use the 1984 and 1993 Surveys of Income and Program Participation (SIPP) to investigate trends in disability prevalence. Their definition is of disability differs mildly from the one Manton and his colleagues use; they define it as difficulty seeing words in a newspaper, lifting and carrying 10 pounds, climbing stairs, or walking a quarter mile. The authors find that prevalence for difficulty in each category declined over the study period. Prevalence ranged from $15.3 \%$ (difficulty seeing words in a newspaper) to $25.8 \%$ (walking $3 / 4$ milie) in 1984 to $11.6 \%$ to $22.3 \%$ for the same categories in 1993, a relative improvement of between $0.9 \%$ and $2.3 \%$ across functions.

Crimmins, Saito, and Reynolds (1997) confirm net decreases in disability among the elderly from 1982 to 1993 despite intermediate fluctuations. They use the Longitudinal Study of Aging (LSOA) (1984, '86, '88, '90) and the National Health Interview Survey (NHIS) (1982 to '93) to track the prevalence of disability over time. They focus solely on the prevalence of disability in the $70+$ population. Overall prevalence of disability in the NHIS (defined as inability to perform personal or routine care) in 1982 was $21.1 \%$ and in 1993 was $19.5 \%$. In the LSOA, they find that the percent disabled (defined as an inability to perform an ADL without aid) in 1984 , ' 86 , ' 87 , and '90 was $18.8,21.6,21.3$, and $20.5 \%$ respectively. Although no visually striking trend exists in the plotted data sets, statistical analysis reveals a slight decrease in disability in 
the pooled population (controlling for age and sex). These decreases are more likely in the activities of IADLs (routine care) as opposed to ADLs (personal care). Hazard models applied to this data reveal that rates of transition to disability and out of disability improved over time: incidence of disability decreased $16 \%$ from 1948 -' 86 to 1988 -' 90 .

Using NHIS 1970, ' 80 , and '90 data and an alternate definition of disability than the one we rely on in this paper (any limitation in usual activity over the past 12 months), Crimmins, Saito, and Ingegneri (1997) find fluctuations in long-term disability prevalence. Institutionalization rates have declined for most ages, but have stayed the same or increased above age 80 . Long-term disability increased for the $60+$ population from 1970 to 1980, but decreased from 1980 to 1990. For example, males aged 65-69 in 1970,1980 , and 1990 had disability rates of $37.84,43.68$, and $39.39 \%$ respectively, and for females the rates were $30,36.2$, and $30.56 \%$. These prevalence figures are subject to assumptions made about the effects of question wording changes after 1982.

Crimmins, Saito, and Ingegneri (1997) also investigated the relative contributions to life expectancy of disability-free and disabled years. They use the NHIS definition of years of active life expectancy: the "years when an individual's health does not affect ability to perform normal activities of life including both major and secondary activities." At age 65, total life expectancy increased from 1970 to 1990 . (Mortality declines from 1980 to 1990 was $1 / 3$ of that from 1970 to 1980 for females and $3 / 4$ of that for males.) But the proportion of that increase that was due to disability free years was small. From 1970 to 1980 , disability-free life expectancy did not increase, but there was a slight increase from 1980 to 1990. McKinlay et al. (1989) also find that disability-free life expectancy has decreased for newborns and middle-aged women from 1964 to 1985 . These findings 
do not entirely support the hypothesis by Fries (1980) that active-life span is increasing faster than total life span.

Freedman et al. (2004) represents the most comprehensive work on documenting disability trends in the elderly population. This report presents the summary view of a large group of distinguished researchers on elderly disability. The main goal of the Freedman et al. group was to comprehensively explore whether five different nationally representative data sets give the same answer about the direction and extent of disability trends. They also looked at how different definitions of disability led to different trend estimates. They summarize their conclusions as follows:

"Although the evidence was mixed for the 1980s and it is difficult to pinpoint when in the 1990s the decline began, during the mid- and late $1990 \mathrm{~s}$, the panel found consistent declines on the order of $1 \%-2.5 \%$ per year for two commonly used measures in the disability literature: difficulty with daily activities and help with daily activities. Mixed evidence was found for a third measure: the use of help or equipment with daily activities."

As we note above, there has been one attempt to use statistical decompositions to measure the extent to which changes in chronic disease explain trends in elderly disability. Using National Health Interview Survey data from 1984 and 1994, Freedman and Martin (2000) find that upper and lower body limitations declined over that decade. Using these same data, Freedman and Martin find that, though the prevalence of many prominent chronic diseases rose over the same period, the prevalence of disability among those with chronic disease fell. ${ }^{\dagger}$

\footnotetext{
${ }^{\dagger}$ Freedman and Martin (2000) rely on linear regression-based methods to decompose rates of disability growth. The validity of these methods depend strongly on the validity of the linearity assumption, and on the low prevalence of jointly occurring chronic diseases.
} 


\subsection{Data and Methods}

We analyzed data from the $1982,1984,1989,1994$, and 1999 waves of the NLTCS in combination with data from the same years of the NHIS. The NLTCS is a nationally representative longitudinal survey of civilians aged 65 and above that was designed to investigate the functional status of the over-65 population. The survey sample consists of approximately 20,000 respondents per survey year and is composed of living members of the longitudinal component plus the addition of people who have turned 65 since the previous wave was completed. The survey asks detailed questions that assess the respondents' functional status as defined by ADLs and IADLs. The specific questions ask whether the respondent required help or used specialized equipment to perform each of the activities. A screener questionnaire administered to the full sample was used to identify the chronically disabled respondents, who subsequently received a second, detailed survey. The noninstitutional detailed questionnaire asked about the presence of a list of chronic diseases. Since the questions regarding health status were not found on the screener questionnaire administered to the full sample, we were unable to estimate the likelihood of disability associated with chronic illness from the NLTCS alone and were obliged to use NHIS data as well. We defined three disability categories: (1) one or more IADL or ADL impairment; (2) one or more IADL impairment only (no ADL impairments) (3) one or more ADL impairment. The NLTCS questions remained unchanged across the time period we studied.

The NHIS is a cross-sectional survey that contains information on the health of a nationally representative sample of the U.S. noninstitutionalized population. Though the list of medical conditions in the NHIS is extensive, any given participant is only asked a 
subset of the total questions drawn from one of six randomly assigned questionnaires.

Each of the six questionnaires is tailored towards a particular organ-system (e.g.

musculoskeletal and cardiovascular). The exception among the conditions considered was overweight, which we calculated directly from weight and height measurements conducted on all survey respondents. As a result, the sample size for the overweight variable is six times that of the other conditions under consideration. Sample weights were adjusted accordingly. The survey was redesigned in 1982 and 1997 to the extent that internally consistent estimates of morbidity can be constructed between these years. To best approximate the 1999 disease prevalence, we age-standardized 1996 NHIS data to the 1999 Census, on the assumption that the disease prevalence did not change significantly between 1996 and 1999 for any given age.

We investigated a set of chronic medical conditions that are prevalent among the elderly and are commonly associated with impaired functional status (Freedman and Martin 2000; Miller RR 2004). They include arthritis, diabetes, hypertension, stroke, heart disease, overweight, and chronic obstructive pulmonary disease (COPD). COPD is a syndrome characterized by airflow obstruction due to emphysema or chronic bronchitis. Being overweight is defined as having a body mass index greater than or equal to 25 .

The NHIS dataset before the 1997 redesign contained only general assessments of disability and thus did not allow for detailed assessment of functional impairment. The NLTCS, in contrast, is particularly well suited for sophisticated evaluation of disability. However, the NLTCS alone was not sufficient for our analysis due to the fact that the screener questionnaire did not include health status questions. 
We addressed this problem by combining the two datasets using Bayes' rule. We calculated the probability of being functionally disabled given the presence of each chronic illness, say $C$, using a formula with three terms: the probability of having the illness given disability $(D)$, the probability of being disabled (the population disability prevalence), and the probability of having the illness (the population disease prevalence). Bayes' rule implies:

$$
P[D \mid C]=\frac{P[C \mid D] P[D]}{P[C]}
$$

The term on the left is our desired result, the prevalence of disability among individuals suffering from condition $C$. For any given year and medical condition, the two terms in the numerator on the right are easily calculated from the NLTCS dataset. The term in the denominator is estimated from NHIS data.

Estimates were calculated with sample weights to adjust for complex survey design and age-standardized to the 1999 Census. The NLTCS public use files contained neither clustering nor stratification variables which precluded the estimation of standard errors accurately corrected for survey design. Spillman (2002) showed that the survey design effect was largest in 1984 when it was equal to 1.4. We applied this conservative design effect estimate to our calculated standard errors to account for the complex survey design of the NLTCS. We also age-standardized to the 1999 Census to control for changes in the age-composition of the population.

\subsection{Decomposing Disability Trends}


In this section, we briefly describe our decomposition of disability trends into two partsthat attributable to changes in the prevalence of chronic disease, and that attributable to changes in the prevalence of disability among those with chronic disease. Our aim is to determine the extent to which age-specific trends in disability can be explained by explained by observed trends in chronic health. Let $D_{t}$ be a dummy variable indicating disability and let $C_{t}$ be a dummy indicating chronic illness at time t. A basic law of probability allows us to decompose the probability (or equivalently, prevalence) of disability into one part attributable to the chronically ill population, and another part attributable to the non-chronically ill population:

$P\left[D_{t}\right]=P\left[D_{t} \mid C_{t}=1\right] P\left[C_{t}=1\right]+\{$ part attributable to non-chronically ill pop. $\}$

Using equation (2), we can write the change in disability prevalence between $t-1$ and $t$, $\Delta P\left[D_{t}\right]$, as follows:

$$
\begin{aligned}
\Delta P\left[D_{t}\right]= & \Delta P\left[D_{t} \mid C_{t}=1\right] P\left[C_{t}=1\right]+P\left[D_{t} \mid C_{t}=1\right] \Delta P\left[C_{t}=1\right] \\
& +\{\text { change attributable to non-chronically ill pop. }\}
\end{aligned}
$$

Each term in (2) contributes two terms to (3): a term that reflects the change due to a change in the prevalence of chronic conditions, and a term that reflects the change due to a change in the probability of disability among those with chronic conditions. In Appendix A, we extend this framework to account for changes in multiple chronic conditions. 


\subsection{Results}

The prevalence of chronic disability among the noninstitutional U.S. elderly declined between 1982 and 1999 (see Table 1). The prevalence of IADL disability diminished by $45 \%$, whereas ADL disability declined by $9 \%$.

The age-adjusted prevalence of selected diseases over the same period is displayed in Figure 1. Between 1982 and 1999, the condition with the largest change (of the set we examined) was overweight, which increased by 10.4 percentage points. The prevalence of arthritis declined by 3.0 percentage points; the prevalence of heart disease declined by 2.6 percentage points; and the prevalence of hypertension declined by 3.3 percentage points. There were also small increases in the prevalence of stroke, chronic obstructive pulmonary disease (COPD), and a 1.1 percentage point increase in the prevalence of diabetes.

Figures 2-4 shows trends in the (age-adjusted) prevalence of disability among elderly individuals with a chronic disease. Each figure corresponds to a different definition of disability. Figure 2 shows trends in the prevalence of individuals with either an ADL or an IADL disability. Figure 3 shows trends in IADL disability, and Figure 4 shows trends in ADL disability. Table 2 summarizes these figures by showing percentage point changes between 1982 and 1999 in disability prevalence among the chronically ill.

Figure 2 shows that Arthritis, overweight, and heart disease were associated with statistically significant changes in the likelihood of being disabled between 1982 and 1999. Among respondents who suffered from arthritis there was a $19 \%$ (5.8 percentage 
point) drop in the likelihood of functional impairment during the 1990s after a decade with no change. Overweight elderly were $30 \%$ less likely to be disabled in 1999 than they were in 1982. The disabling effect of heart disease remained unchanged during the 1980s, subsequently dropping by $24 \%$ during the 1990s. Diabetes and stroke-associated disability prevalence ended the period essentially unchanged. Point estimates for COPDand hypertension-associated disability rates dropped $28 \%$ and $10 \%$ respectively between 1982 and 1999 with the bulk of the change taking place between 1989 and 1999, though neither change was statistically significant.

Figures 3 and 4 show trends in IADL-only and ADL disability. In general, IADL disability declined more sharply than ADL disability between 1982 and 1999. Arthritis, hypertension, COPD, overweight, and heart disease were associated with substantial declines in IADL disability $(\mathrm{p}<.01)$. In all cases, the decline approached $50 \%$ and was greater during the 1990s than during the 1980s. Point estimates of the disabling effect of diabetes declined $25 \%$ but the change was not statistically significant. In contrast, stroke sufferers experienced no significant change in IADL impairment. Among the seven conditions evaluated, only overweight was associated with a statistically significant decline $(\mathrm{p}<.05)$ in ADL disability between 1982 and 1999, a decrease of about 20\%. The probability of ADL disability dropped about $20 \%(\mathrm{p}<.10)$ among respondents with heart disease during the 1990s. Point estimates for arthritis and COPD-associated ADL disability declined $12 \%$ and $18 \%$ respectively during the 1990 s, though the changes were not statistically significant.

Finally, Table 3 shows the results from our disability decomposition. The first three columns show the portion of disability trends that is attributable to changes in the 
prevalence of each chronic condition $-P\left[D_{t} \mid C_{t}=1\right] \Delta P\left[C_{t}=1\right]$. The second three columns show the portion of disability trends that is attributable to changes in the prevalence of disability among those with each chronic conditions$\Delta P\left[D_{t} \mid C_{t}=1\right] P\left[C_{t}=1\right] . \quad$ It is clear from this table that the well-known decline in disability prevalence masks important increases in disability caused by increasing prevalence of chronic disease, especially in overweight and diabetes. Absent any other changes, prevalence trends in these chronic conditions alone would have increased disability rates by over 200 cases per 10,000 elderly individuals between 1982 and 1999 . The modest prevalence declines in arthritis, heart disease, and hypertension are responsible for a decrease in about 264 disability cases per 10,000 population over the same period. Stroke and COPD prevalence also rose modestly, accounting for an increase of 27 cases per 10,000 population.

By contrast, the prevalence of disability among those with a chronic illness declined sharply for every disease we examined, except stroke. The biggest declines were due to declining disability among elderly patients with arthritis (290 cases per $10,000)$, heart disease (176.8 cases per 10,000), and overweight (179 cases per 10,000). To some extent, these number overstate the true decline because of double counting - $\mathrm{a}$ substantial number of people were both overweight and had heart disease. Nevertheless, this table suggests substantial declines in disability prevalence among the chronically ill. In nearly every case, though, declines were sharpest in the prevalence of milder IADLtype disabilities, rather than in ADL-type disabilities..

\subsection{Discussion}


We did not find evidence of a downward trend in disease prevalence that could be used to justify the broad decline in elderly disability rates that occurred between 1980 and 2000. Moderate declines in self-reported heart disease and hypertension took place between 1984 and 1999, which probably reflect increasingly aggressive treatment of heart disease risk factors such as hypertension and dyslipidemia over the last two decades (Fries, Cutler). A substantial increase in the prevalence of overweight was observed over this time period, a well-established and troubling trend that is likely to oppose reductions in chronic illness and disability rates in the future. Earlier detection of illness, which has been proposed as an explanation for declines in measured morbidity-associated disability rates, would result in greater disease prevalence estimates, all other things being equal, and could therefore have masked declines in elderly disease burden.

Measured disability did in fact decline between 1982 and 1999 among the U.S. over-65 population and our estimates corroborate the findings of other investigators which imply that IADL disability improvements were the main source of advancements in elderly functioning. More severe functional impairment, as measured by ADLs, improved only modestly. Other studies of the same time period have encountered no improvement in ADL disability or even a moderate increase. As mentioned previously, the average number of ADLs actually increased (Spillman, 2003). Furthermore, according to our evidence, an acceleration of elderly functional improvement took place during the 1990 s as compared to the 1980 s.

Trends in the disabling effects of individual chronic illnesses differed for the two categories of disability measurement. Chronic disease-associated IADL disability declined broadly for nearly all diseases and the magnitude of the drop was comparable in 
all cases - between 40 and $60 \%$. Several theories that have been proposed to explain reductions in disability prevalence are commensurate with a broad IADL disability decline. Spillman (2003) suggested that observed IADL improvements might reflect changes in the environment that would make such tasks as managing money and grocery shopping easier regardless of disability status, a theory that aligns well with our results. Internet shopping, amplifying devices for phones, and street ramps are examples of changes in the environment that could result in increased facility with IADL tasks. The changing socioeconomic composition has also been employed as an explanation for lower disability rates. A clear correlation exists between educational level, income level, and lower disability status, and trends toward higher levels of education and income among U.S. elderly have been documented. The manner by which education and income enhance functioning remains to be demonstrated but plausible explanations include the links between education and health-improving behaviors and between income and better healthcare. Alternate theories that may help to explain a broad IADL disability decline include healthier behavior, such as reduced smoking rates, which have been correlated with better functioning (Cutler 2001), decreased lifetime exposure to infectious disease, the doubling of the share of persons receiving cataract surgery, and increased use of aids/equipment such as walkers, handrails, and walk-in showers.

Although adoption of aids and equipment has been documented and is an intuitive explanation for reduced disability, in this study disability was measured as needing help or using special equipment, in which case the use of aids in place of human help should not register as a functional improvement and therefore cannot explain the trend in the data. This is given support by the fact that the prevalence of "human help only" 
disability declined faster over this period than the disability rate reflected by the "either help or equipment" measure. For some tasks, such as bathing, the rate of disability measured by the use of special equipment rose in contrast to the rate measured by the utilization of human help. It is worth noting that certain technological advancements, such as automatic electronic bill payment, which are likely to have improved elderly functioning over this period, would not have been scored as use of special equipment. These types of environmental changes would more likely be associated with IADL activities than ADL activities which require more conspicuous mechanical aids.

In contrast to IADL disability, change in the probability of having ADL disability given a chronic illness was narrow. The only statistically significant change observed between 1982 and 1999 was a 20\% decline in overweight-associated ADL disability prevalence. Unfortunately, the simultaneous $25 \%$ rise in the prevalence of overweight more than compensated for this improvement, with a net increase in ADL disability among the overweight elderly. In contrast, the decline IADL disability among the overweight over this time period was greater than the increase in IADL disability that resulted from the larger prevalence of overweight. Heart disease, arthritis, and COPDassociated ADL disability prevalence estimates declined during the 1990s, though only the heart disease change was statistically significant.

In contrast to the IADL measure, the narrowness and modesty of ADL disability declines suggest that different factors may be responsible. In some cases, earlier detection at less severe stages of illness may have contributed to measured declines in disability. A closer look at the particular illnesses associated with observed improvements in ADL disability allows us to suggest possible explanations. In both of 
the studies mentioned in the introduction, evidence of functional improvements among arthritics was presented, though in the case of ADL disability Crimmins et al. found deterioration between 1984 and 1994 . We calculated gains in functioning among elderly arthritics during the 1990s with little to no change during the 1980s. The treatment of arthritis has undergone changes over recent decades that may have contributed to the observed improvements. For example, the number of joint replacement surgeries approximately doubled from the 1980s to the 1990s (Cutler, 2001). The use of nonsteroidal anti-inflammatory drugs (NSAIDs) and disease modifying antirheumatic drugs (DMARDs), medications commonly used for the treatment of arthritic symptoms, has risen dramatically since the 1970s. The treatment of heart disease and its risk factors has become much more aggressive over this time period as well. Improved functioning among COPD sufferers may be attributable to reduced smoking rates, newer portable supplemental oxygen tanks, the introduction of and growing enrollment in specialized pulmonary rehabilitation centers, and an increased emphasis on better conditioning and exercise in treatment strategies.

\subsection{Conclusion}

The decline in disability rates among U.S. elderly is a widely accepted finding. The implications for this decline with respect to forecasting future patterns of Medicare and Social Security expenditures remain unclear and depend upon which factors are responsible for the improvement. Some have come to the conclusion that the solvency of federal entitlement programs could be preserved if the observed rate of decline in disability were to persist (Singer et al 1998). Evidence of successful disease prevention 
would lend support to this optimistic view. However, it is quite possible that functional improvements may have been the result of technological innovation and increasingly aggressive treatment strategies, both of which could increase, rather than decrease, healthcare expenditures.

Our findings suggest that primary prevention, as reflected in decreased disease prevalence, was not responsible for advances made in elderly functioning between 1980 and 2000. We found a broad decline in less severe forms of disability that is unlikely to have resulted from improved disease management. Instead, these measured improvements in functioning may reflect environmental, technological, and/or socioeconomic changes. Improvements in the more severe forms of disability were modest and were restricted to those suffering from particular illnesses, which make improved and/or more aggressive management a plausible explanation and one that might increase costs should the trend persist. In sum, we find it premature to forecast cost savings based on the lack of disease prevention and the evidence that functional improvements among the most impaired have come at the cost of increasingly aggressive treatment strategies. 


\section{References}

American Lung Association Epidemiology and Statistics Unit. "Trends in Chronic Bronchitis and Emphysea: Morbidity and Mortality." Online publication. http://www.lungusa.org/data/copd/copd1.pdf. Last accessed: September 30, 2002a.

American Lung Association, "Trends in Asthma Morbidity and Mortality January 2001," online publication, http://www.lungusa.org/data/asthma/asthmach chg.html, last accessed September 30, 2002b.

Autor, David and Mark Duggan (2001). "The Rise in Disability Recipiency and the Decline in Unemployment.” Working Paper No. 8336, National Bureau of Economic Research, June. Cambridge, MA: NBER.

Berry, Judy O. and Michael L. Hardman. Lifespan Perspectives on the Family and Disability. Needham Heights, MA: Allyn \& Bacon, 1998.

Bhattacharya J, Currie J. "Youths and Nutritional Risk: Malnourished or Misnourished?" in Risky Behavior Among Youths, J Gruber (ed.), (2001)

Bierman, Arlene S. "Activity Status: The Sixth Vital Sign.” Journal of General Internal Medicine. 16: 785-786, 2001.

Boult, C., M. Altmann, D. Gilbertson, C. Yu, and R.L. Kane. "Decreasing disability in the 21 st century: the future effects of controlling six fatal and nonfatal conditions." American Journal of Public Health. 86(10): 1388-93, 1996.

Bound, John, and Timothy Waidmann (2000). "Accounting for Recent Declines in Employment Rates Among the Working-Aged Disabled.” Working Paper 7975, National Bureau of Economic Research. Cambridge, MA: NBER.

Centers for Disease Control and Prevention. "Trends in the Prevalence and Incidence of Self-Reported Diabetes Mellitus -- United States, 1980-1994." Morbidity and Mortality Weekly Report. 46(43): 1014-8, 1997.

Centers for Disease Control and Prevention. "Arthritis Prevalence and Activity Limitations - United States, 1990." Morbidity and Mortality Weekly Report. 43(24): 433-8, 1994.

Colvez, A., and M. Blanchet. "Disability trends in the United States population 1966-76: analysis of reported causes." American Journal of Public Health. 71(5): 464-71, 1981.

Costa and Steckel "Long-Term Trends in Health, Welfare, and Economic Growth in the United States" In Health and Welfare during Industrialization, ed. R. Floud and R. H. Steckel, 47-89. Chicago: University of Chicago Press for NBER. (1997) 
Crimmins EM (2004) "Trends in the Health of the Elderly," Annual Review of Public Health 25: 79-98.

Crimmins, Eileen M., Yasuhiko Saito, and Dominique Ingegneri (1989). "Changes in Life Expectancy and Disability-Free Life Expectancy in the United States." Population and Development Review 15(2): 235-267.

Crimmins, Eileen M., Yasuhiko Saito, and Sandra L. Reynolds (1997). "Further Evidence on Recent Trends in the Prevalence and Incidence of Disability Among Older Americans From Two Sources: the LSOA and the NHIS." Journal of Gerontology 52B(2): S59-S71.

Freedman VA and Martin LG (1998) "Understanding Trends in Activity Limitations Among Older Americans," American Journal of Public Health 88:1457-1462.

Freedman VA and Martin LG (2000) "Contribution of Chronic Conditions to Aggregate Changes in Old Age Functioning" American Journal of Public Health 90(11):1755-60.

Freedman VA, Crimmins E, Schoeni RF, Spillman BC, Aykan H, Kramarow E, Land K, Lubitz J, Manton K, Martin LG, Shinberg D, Waidmann T. (2004) "Resolving inconsistencies in trends in old-age disability: report from a technical working group" Demography 41(3):417-41.

Fries JF. “Aging, natural death, and the compression of morbidity.” N Engl J Med 1980; 303: $130-35$

Gruenberg, E.M. (1977). “The Failures of Success.” Milbank Quarterly 55: 3-34.

Knoblauch, Bernadette, and Barbara Sorenson. "IDEA's Definition of Disabilities. ERIC Digest E560.” Online publication. http://www.ed.gov/databases/ERIC Digests/ed429396.html. Last accessed September 29, 2002.

Krute, A., and M.E. Burdette. "1972 Survey of disabled and nondisabled adults: chronic disease, injury, and work disability.” Social Security Bulletin. 41(4): 3-17, 1978.

Lakdawalla D., Goldman D, Bhattacharya J, Hurd M, Joyce G, and Panis C., "Forecasting the Nursing Home Population", Medical Care 41(1):8-20 (2003a)

Lakdawalla D., Goldman D, Bhattacharya J, Hurd M, Joyce G, and Panis C., "A Response to the Points by Manton and Williamson on 'Forecasting the Nursing Home Population'”, Medical Care 41(1):28-31 (2003b)

Lakdawalla D, Bhattacharya J, and Goldman D. "Are the Young Becoming More Disabled?" Health Affairs 23(1):168-176 (2004). 
Manton, Kenneth G., Larry Corder, and Eric Stallard. (1993). "Estimates of Change in Chronic Disability and Institutional Incidence and Prevalence Rates in the U.S. Elderly Population from the 1982, 1984, and 1989 National Long Term Care Survey." Journal of Gerontology: Social Sciences 48(4): S153-66.

Manton, Kenneth G., and XiLiang Gu (2001). "Changes in the Prevalence of Chronic Disability in the United States Black and Nonblack Population Above Age 65 from 1982 to 1999." Proceedings of the National Academy of Science 98(11): 6354-9.

Manton, Kenneth G., Larry Corder, and Eric Stallard (1997). "Chronic Disability Trends in Elderly United States Populations: 1982-1994." Proceedings of the National Academy of Science 94: 2593-2598.

Marshak, Laura E., Milton Seligman, and Fran Prezant. Disability and the Family Life Cycle: Recognizing and Treating Developmental Challenges. New York: Basic Books, 1999.

McKinlay JB, McKinlay SM, Beaglehole R. "A review of the evidence concerning the impact of medical measures on recent mortality and morbidity in the United States.” Int J Health Serv. (1989);19(2):181-208

McNeil, John M. "Employment, Earnings, and Disability." Prepared for the $75^{\text {th }}$ Annual Conference of the Western Economic Association International. Vancouver, BC: June 29-July 3, 2000.

Mokdad, Ali H., Earl S. Ford, Barbara A. Bowman, David E. Nelson, Michael M. Engelgau, Frank Vinicor, and James S. Marks. "Diabetes Trends in the U.S.: 1990-1998.” Diabetes Care. 23(9): 1278-83, 2000.

National Heart, Lung, and Blood Institute. "Congestive Heart Failure in the United States: A New Epidemic." Online publication. http://www.nhlbi.nih.gov/health/public/heart/other/CHF.htm. Last accessed September 30, 2002.

Newacheck, Paul W., and Neal Halfon. "Prevalence, Impact, and Trends in Childhood Disability Due to Asthma." Archives of Pediatrics and Adolescent Medicine. 154(3): 287-93, 2000.

Pardes H, Manton KG, Lander ES, Tolley HD, Ullian AD, Palmer H "Effects of Medical Research on Health Care and the Economy.” Science. v. 283 \#5398 (1999) p. 367.

Reynolds, S.L., E.M. Crimmins, and Y. Saito. "Cohort differences in disability and disease presence." The Gerontologist. (1999) 38(5): 578-90. 
Social Security Administration. "Social Security Disability Planner." Online publication. http://www.ssa.gov/dibplan/dqualify4.htm. Last accessed September 29, 2002.

Stone, Deborah A. The Disabled State. Philadelphia: Temple UP, 1984.

Schoeni, Robert F., Vicki A. Freedman, and Robert B. Wallace (2001). "Persistent, Consistent, Widespread, and Robust? Another Look at Recent Trends in Old-Age Disability." Journal of Gerontology, forthcoming.

Stoddard, S., L. Jans, J. Ripple, and L. Kraus. Chartbook on Work and Disability in the United States, 1998. An InfoUse Report. Washington, D.C.: U.S. National Institute on Disability and Rehabilitation Research, 1998.

Songer, Thomas J. "Disability in Diabetes." In Diabetes in America. 2nd ed. National Diabetes Data Group. Washington, DC: US GPO, 1995.

United States Bureau of the Census. "Disability Status of Persons (SIPP)." Online publication. http://www.census.gov/hhes/www/disable/sipp/disstat.html. Last accessed September 30, 2002a.

United States Bureau of the Census. "Americans with Disabilities: 1997 - Table 2." Online publication.http://www.census.gov/hhes/www/disable/sipp/disab97/ds97t2.html. Last accessed September 30, 2002 b.

United States Code. "Social Security Act \$216." Online publication. http://www.law.cornell.edu/socsec/act/0216.htm. Last accessed September 29, 2002.

United States Department of Labor Office of Disability Employment Policy. "How does the Feceral government define 'disability"'? Online publication. http://www.dol.gov/odep/faqs/federal.htm. Last accessed September 29, 2002.

United States Equal Employment Opportunity Commission. "Executive Summary: Compliance Manual Section 902, Definition of the Term Disability." Online publication. http://www.eeoc.gov/docs/902sum.html. Last accessed September 29, 2002.

Waidmann, Timothy, John Bound, and Michael Schoenbaum (1995). "The Illusion of Failure: Trends in the Self-Reported Health of the U.S. Elderly." Milbank Quarterly 73(2): 253-287.

Wolf, Anne, and Graham Colditz (1998). "Current Estimates of The Economic Cost of Obesity in The United States.” Obesity Research 6(2): 97-106. 
Table 1: NLTCS Disability Prevalence Trends

\begin{tabular}{|l|rrrrr|}
\hline & 1982 & 1984 & 1989 & 1994 & 1999 \\
\hline \hline Nondisabled & $74.1 \%$ & $74.3 \%$ & $74.6 \%$ & $77.7 \%$ & $79.7 \%$ \\
IADL only & $5.7 \%$ & $6.1 \%$ & $4.9 \%$ & $4.4 \%$ & $3.2 \%$ \\
1 or 2 ADLs & $6.8 \%$ & $6.8 \%$ & $7.0 \%$ & $6.0 \%$ & $6.1 \%$ \\
3 or 4 ADLs & $3.0 \%$ & $3.1 \%$ & $3.8 \%$ & $3.3 \%$ & $3.6 \%$ \\
5 or 6 ADLs & $3.7 \%$ & $3.4 \%$ & $3.1 \%$ & $2.9 \%$ & $3.0 \%$ \\
Institutionalized & $6.7 \%$ & $6.4 \%$ & $6.6 \%$ & $5.6 \%$ & $4.3 \%$ \\
\hline
\end{tabular}

Note: Age standardized to 1999 Census

Table 2: Change in Disability Prevalence Among the Chronically Ill—1982-1999

\begin{tabular}{|l|ccc|}
\hline & \multicolumn{3}{|c|}{ Disability Definition } \\
Condition & Any Disability & IADL & ADL \\
\hline \hline Arthritis & $-5.8 \%$ & $-3.6 \%$ & $-2.2 \%$ \\
COPD & $-17.2 \%$ & $-8.8 \%$ & $-8.1 \%$ \\
Diabetes & $-1.0 \%$ & $-2.6 \%$ & $1.5 \%$ \\
Heart & & & \\
Disease & $-6.6 \%$ & $-3.8 \%$ & $-3.0 \%$ \\
Hypertension & $-2.7 \%$ & $-3.2 \%$ & $0.6 \%$ \\
Overweight & $-4.3 \%$ & $-2.1 \%$ & $-2.2 \%$ \\
Stroke & $0.5 \%$ & $0.1 \%$ & $0.1 \%$ \\
\hline
\end{tabular}

Note: Age standardized to 1999 Census

Table 3: Disability Decomposition

\begin{tabular}{|l|ccc|ccc|}
\hline & \multicolumn{3}{|c|}{ Change Attributable to } & \multicolumn{3}{c|}{ Change Attributable to Disability } \\
Condition & All Disability & ADL & IADL & All Disability & ADL & IADL \\
\hline \hline Arthritis & -99.6 & -73.5 & -26.3 & -289.7 & -109.3 & -181.7 \\
COPD & 11.3 & 8.1 & 3.1 & -140.0 & -66.2 & -71.6 \\
Diabetes & 55.5 & 43.6 & 12.6 & -8.9 & 13.0 & -22.1 \\
Heart & & & & & & \\
Disease & -71.0 & -51.6 & -19.4 & -176.8 & -79.5 & -100.4 \\
Hypertension & -93.4 & -67.6 & -26.0 & -105.2 & 22.9 & -125.6 \\
Overweight & 146.8 & 109.6 & 37.9 & -179.9 & -91.4 & -89.0 \\
Stroke & 15.7 & 14.2 & 1.9 & 3.3 & 0.7 & 0.5 \\
\hline
\end{tabular}

Note: Age standardized to 1999 Census. Each entry is expressed in number of cases per 10,000 population. 
Figure 1: Age Adjusted Disease Prevalence Trends

Panel A: Diabetes, Stroke, Heart Disease, Hypertension

$$
\mathrm{P}[\mathrm{C}] \text { (Age Adjusted) }
$$

Diabetes, Stroke, Heart Disease, or Hypertension
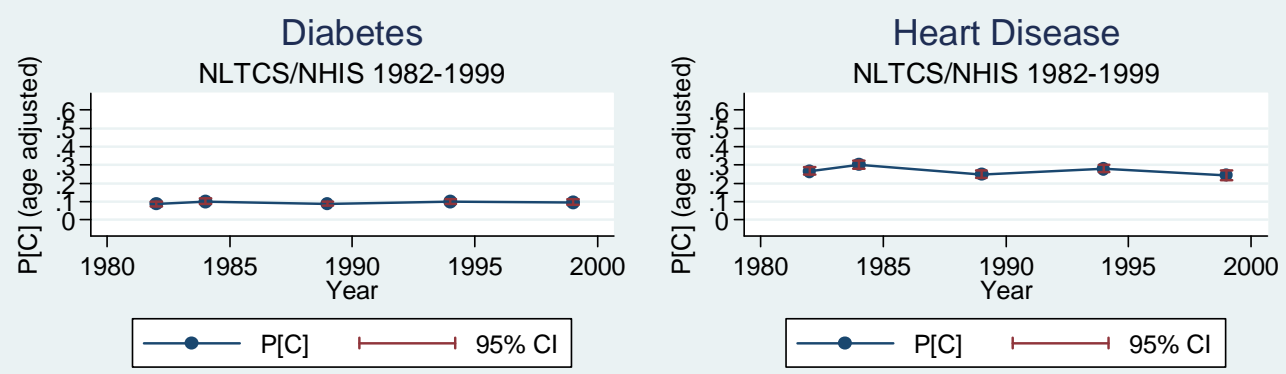

Hypertension
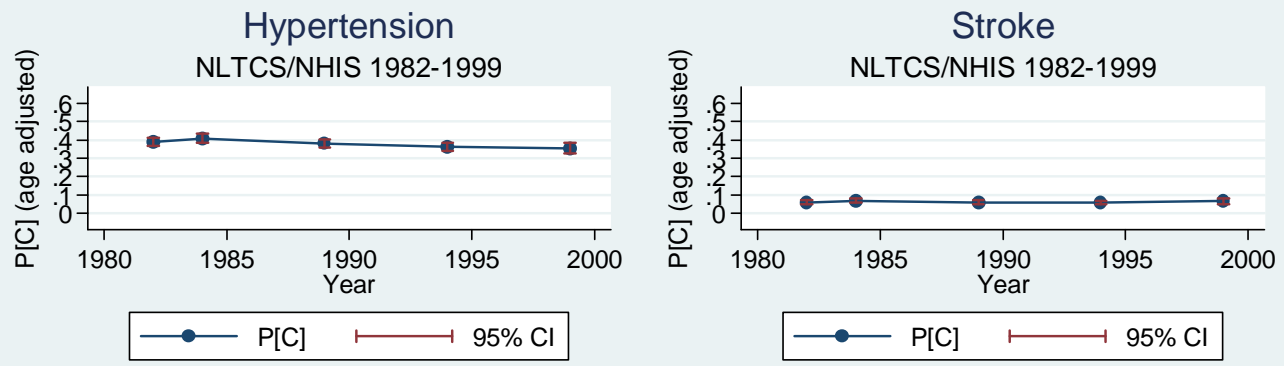

Panel B: Arthritis, COPD, Overweight

$\mathrm{P}[\mathrm{C}]$ (Age Adjusted)

Arthritis, COPD, or Overweight
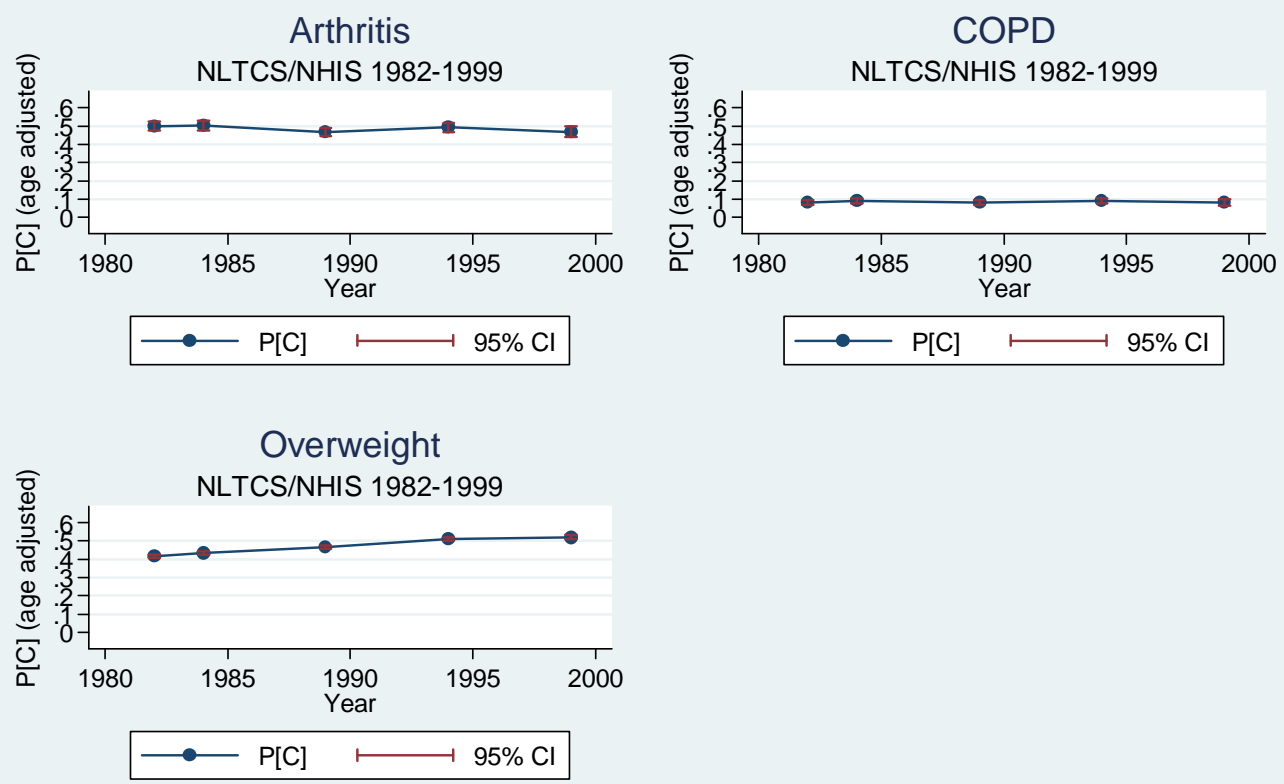
Figure 2: Age-Adjusted Disability Trends Among the Chronically Ill (Any Disability)

Panel A: Diabetes, Stroke, Heart Disease, Hypertension

$$
\text { P[D | C ] (Age Adjusted) }
$$

Diabetes, Stroke, Heart Disease, or Hypertension
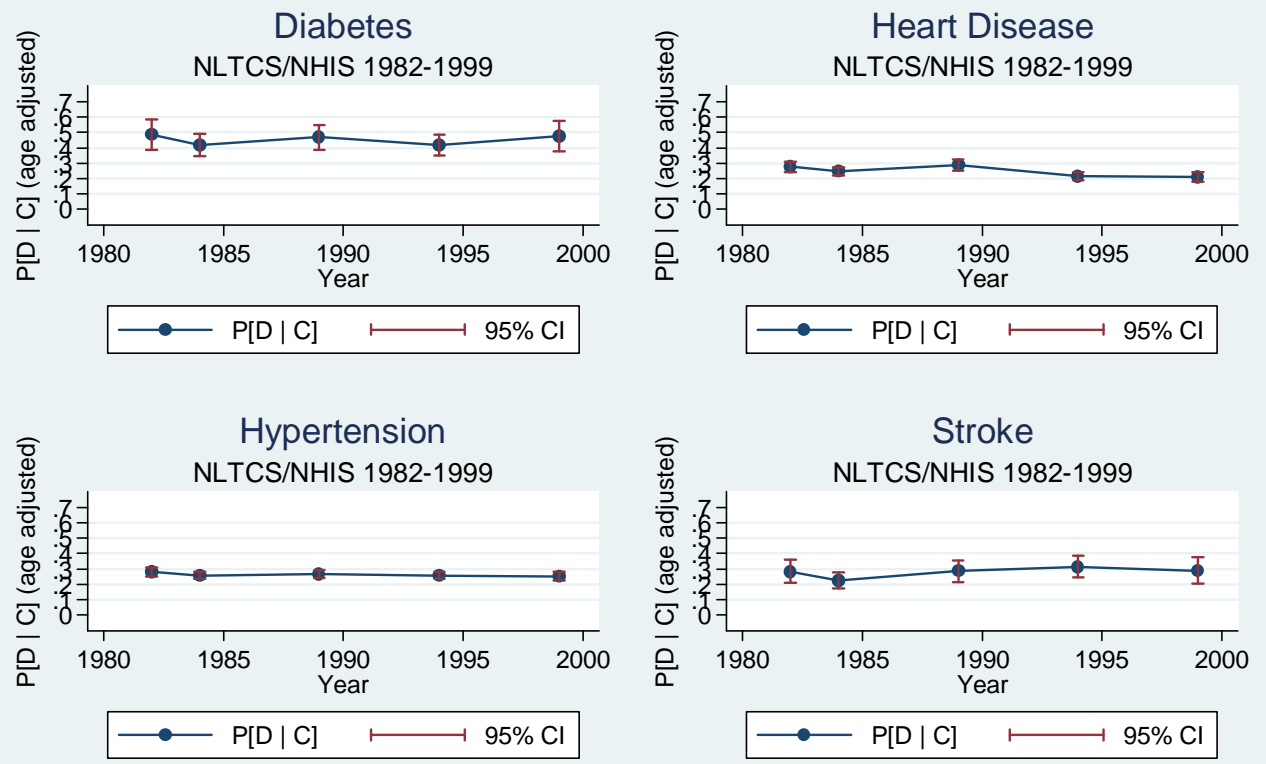

Panel B: Arthritis, COPD, Overweight

$\mathrm{P}[\mathrm{D} \mid \mathrm{C}]$ (Age Adjusted)

Arthritis, COPD, or Overweight
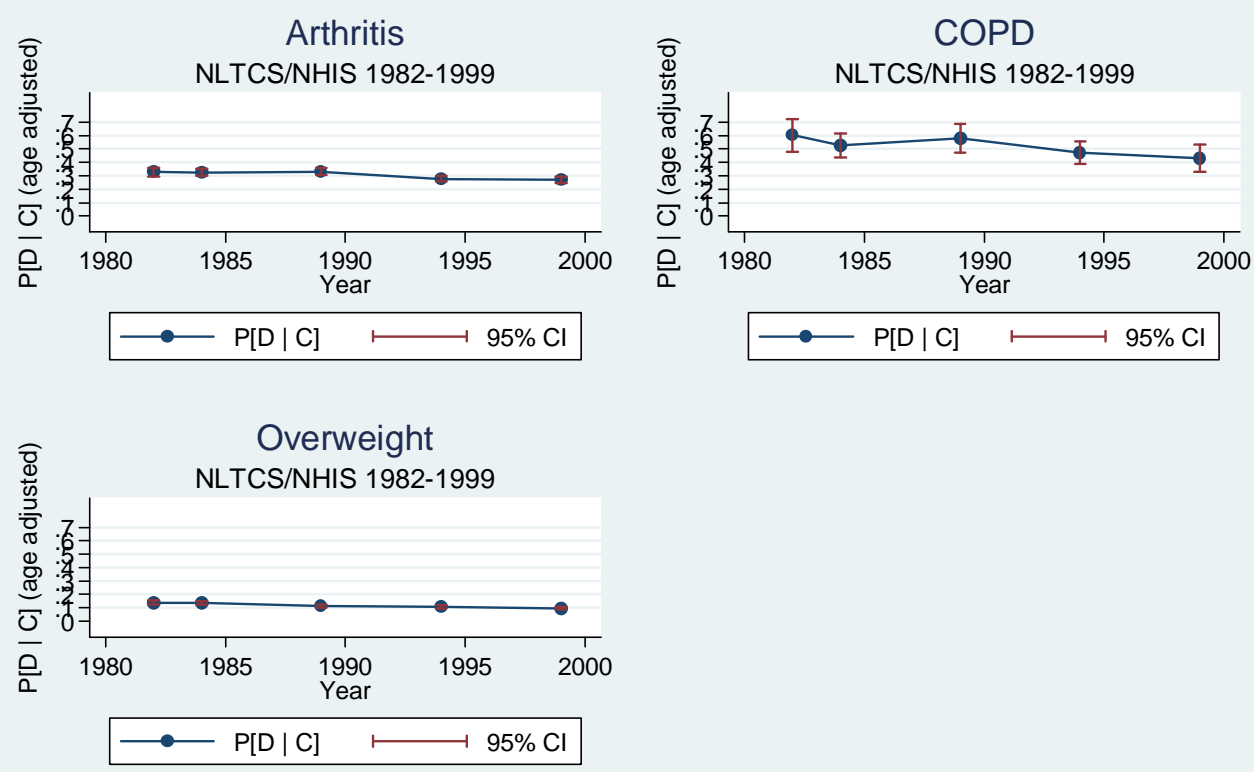
Figure 3: Age-Adjusted Disability Trends Among the Chronically Ill (IADL Disability)

Panel A: Diabetes, Stroke, Heart Disease, Hypertension

$$
\text { P[1+ IADL | C] (Age Adjusted) }
$$

Diabetes, Stroke, Heart Disease, or Hypertension
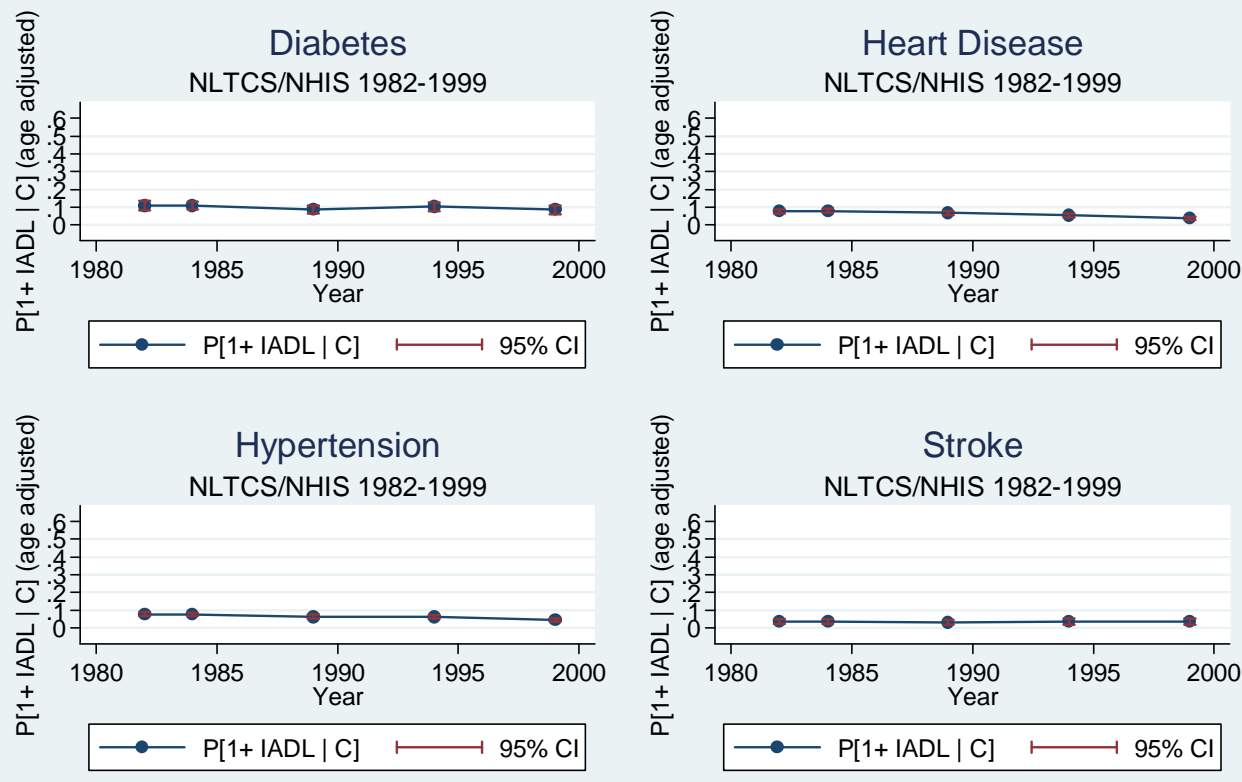

Panel B: Arthritis, COPD, Overweight

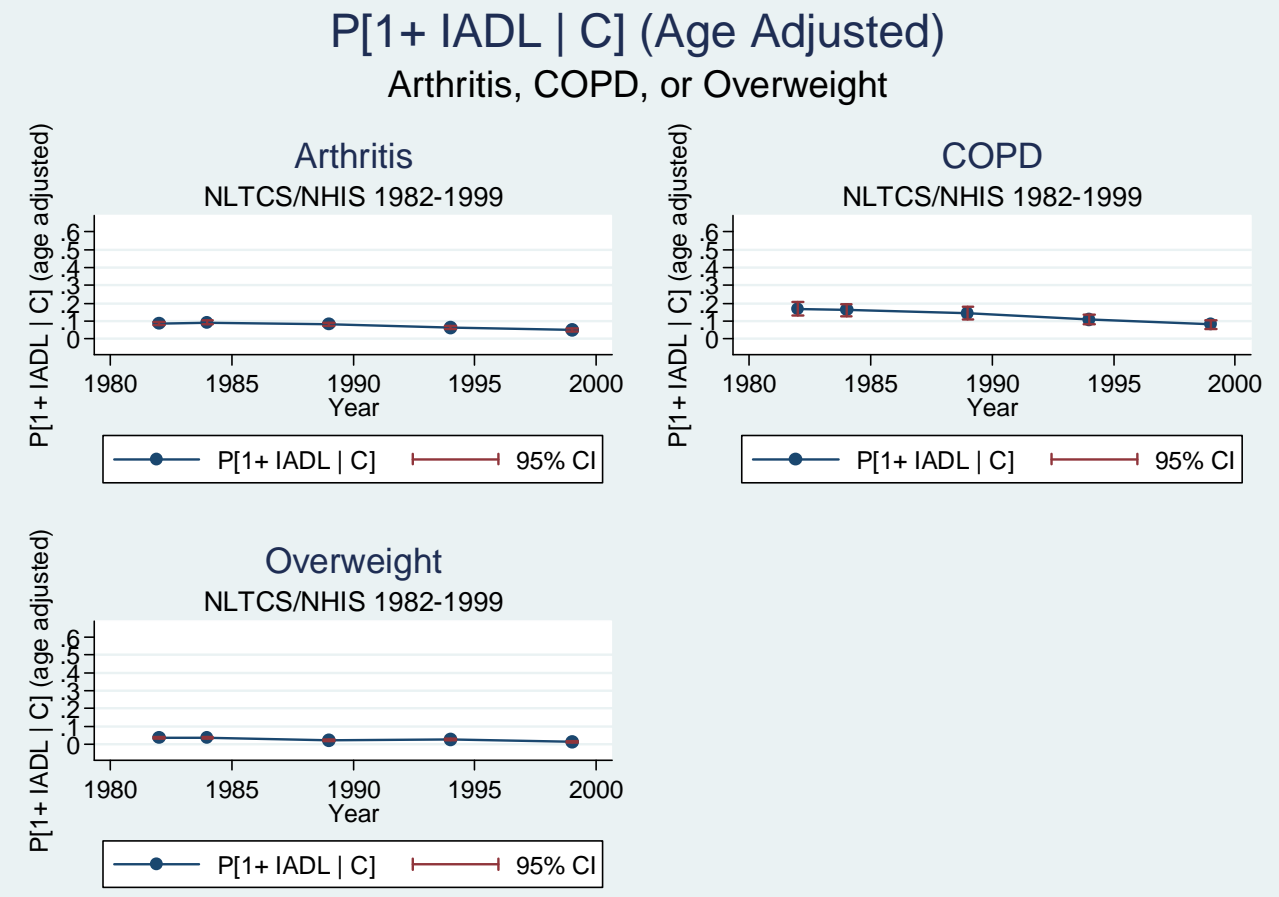


Figure 4: Age-Adjusted Disability Trends Among the Chronically Ill (ADL Disability)

Panel A: Diabetes, Stroke, Heart Disease, Hypertension

$$
\mathrm{P}[1+\mathrm{ADL} \mid \mathrm{C}] \text { (Age Adjusted) }
$$

Diabetes, Stroke, Heart Disease, or Hypertension
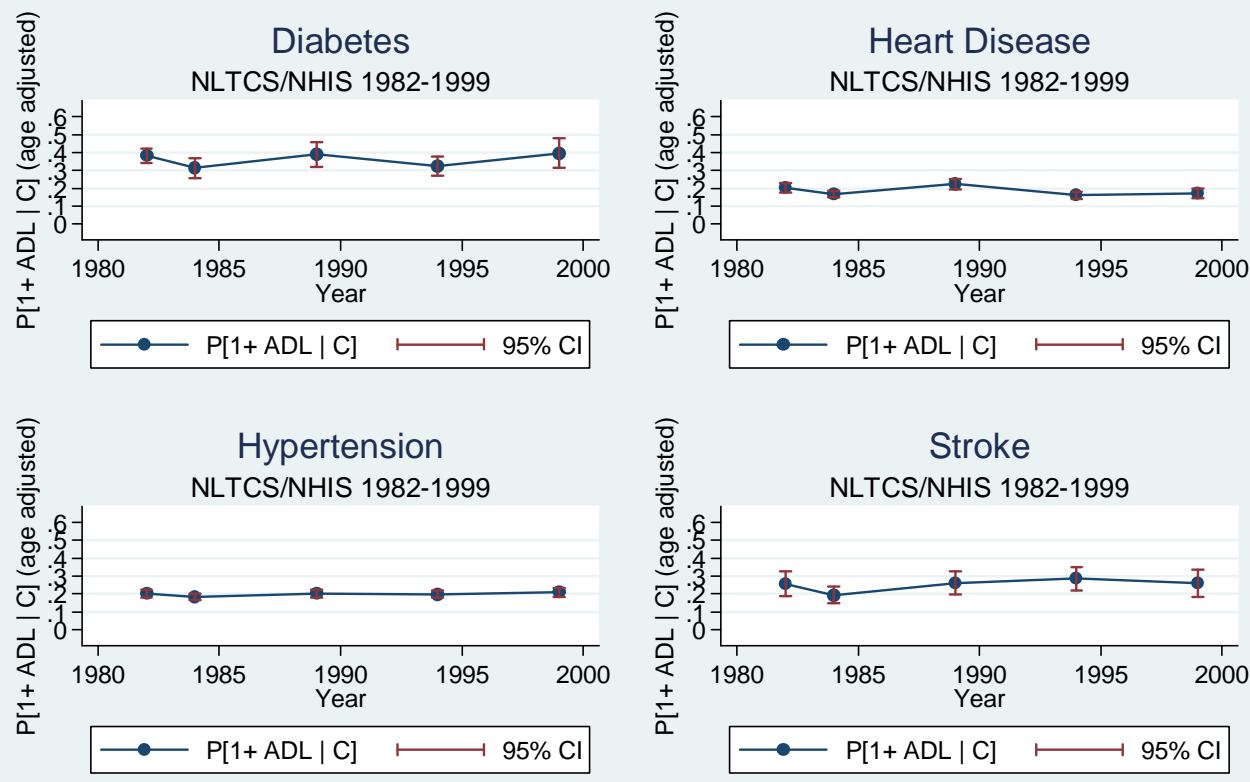

Panel B: Arthritis, COPD, Overweight

\section{$P[1+A D L \mid C]$ (Age Adjusted) \\ Arthritis, COPD, or Overweight}
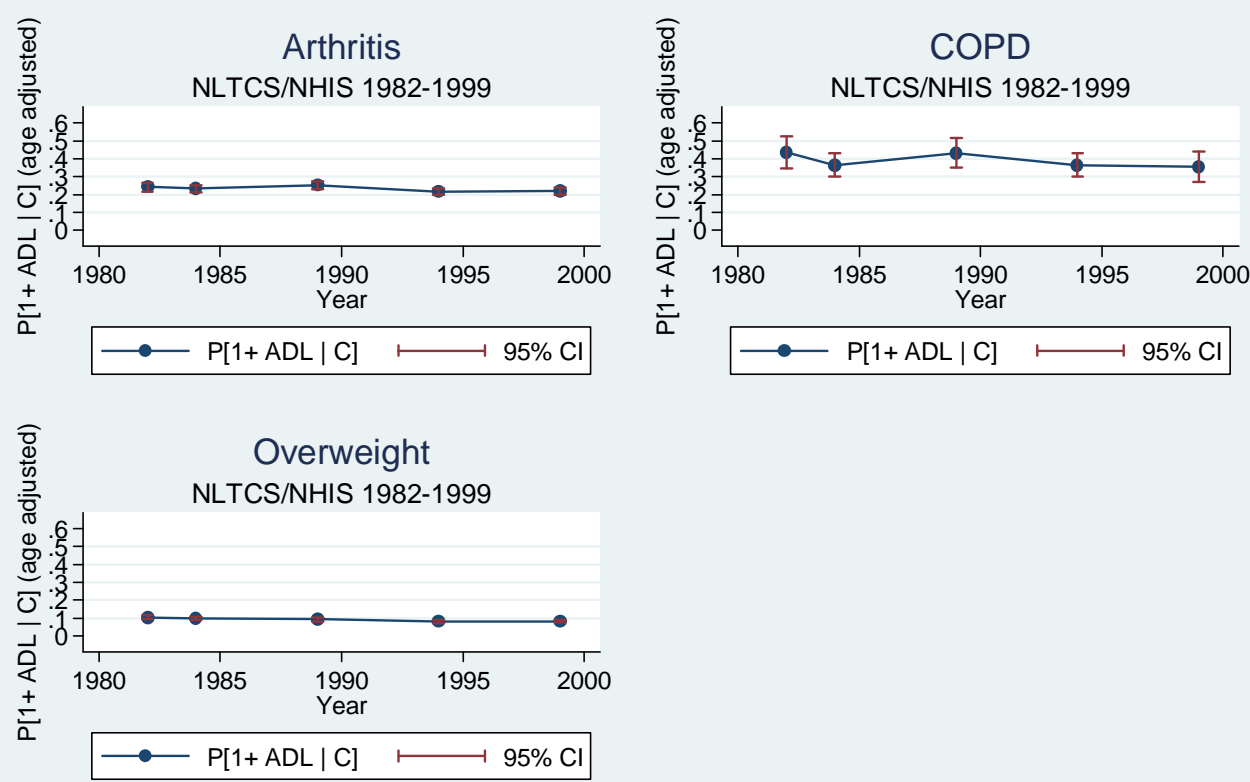


\section{Appendix A: Decomposing Causes of Changes in Disability}

The aim here is to determine the extent to which trends in disability can be explained by explained by observed trends in chronic health. For each person $i$ (suppressed for clarity), let $D_{t}$ be a dummy indicating self-reported disability at time $t$ and let $C_{t}=\left\{C_{1 t}, C_{2 t}, \ldots, C_{K t}\right\}$ be a vector of dummy variables, each of which indicates whether a particular chronic condition is present and observed (by the econometrician) at time $t$. For illustration, consider just the first chronic condition, $C_{1 t}$. The prevalence of disability can be written as follows:

$$
P\left[D_{t}\right]=P\left[D_{t} \mid C_{1 t}=1\right] P\left[C_{1 t}=1\right]+P\left[D_{t} \mid C_{1 t}=0\right] P\left[C_{1 t}=0\right]
$$

The proportion of the people with disabilities that is attributable to people with $C_{1 t}$ is simply the first of two terms in the previous equation, while the second term is the proportion of the people with disabilities that is attributable to people without $C_{1 t}$ (though these people may have other chronic conditions, or may report being disabled due to public policy, accidents, or other health trends). Using equation (A-1), we can decompose the change in disability prevalence between t-1 and t, $\Delta P\left[D_{t}\right]$, as follows:

$$
\begin{aligned}
& \Delta P\left[D_{t}\right]=\Delta P\left[D_{t} \mid C_{1 t}=1\right] P\left[C_{1 t}=1\right]+P\left[D_{t} \mid C_{1 t}=1\right] \Delta P\left[C_{1 t}=1\right] \\
& +\Delta P\left[D_{t} \mid C_{1 t}=0\right] P\left[C_{1 t}=0\right]+\Delta P\left[D_{t} \mid C_{1 t}=0\right] P\left[C_{1 t}=0\right]
\end{aligned}
$$

Each term in (A-1) contributes two terms to (A-2): a term that reflects the change due to a change in the prevalence of the condition and a term that reflects the change due to a change in the probability of disability among those with the condition.

Now, let $E_{t}$ be the portion of disability prevalence that can be explained by the chronic conditions in $C_{t}$ when they are observed singly. Define $E_{t}$ as a generalization of the first term in (A-1):

$$
E_{t}=\sum_{k=1}^{K} P\left[D_{t} \mid C_{k t}=1\right] P\left[C_{k t}=1\right]
$$

Let $\Delta E_{t}$ be the portion of the change in disability prevalence between $t-1$ and $t$ that can be explained by the chronic conditions in $C_{t}$ :

$$
\begin{array}{cc}
\Delta E_{t}= & \Delta P\left[D_{t} \mid C_{1 t}=1\right] P\left[C_{1 t}=1\right]+P\left[D_{t} \mid C_{1 t}=1\right] \Delta P\left[C_{1 t}=1\right] \\
& +\Delta P\left[D_{t} \mid C_{2 t}=1\right] P\left[C_{2 t}=1\right]+P\left[D_{t} \mid C_{2 t}=1\right] \Delta P\left[C_{2 t}=1\right] \\
& \vdots \\
& +\Delta P\left[D_{t} \mid C_{K t}=1\right] P\left[C_{K t}=1\right]+P\left[D_{t} \mid C_{K t}=1\right] \Delta P\left[C_{K t}=1\right]
\end{array}
$$


To show how $E_{t}$ and $P\left[D_{t}\right]$ are related, consider the case where the presence of only two chronic conditions are observed (that is, $K=2$ ). In that case, the proportion of disability attributable to each of the conditions can be decomposed as follows:

$$
\begin{aligned}
& P\left[D_{t} \mid C_{1 t}=1\right] P\left[C_{1 t}=1\right]=P\left[D_{t} \mid C_{1 t}=1, C_{2 t}=1\right] P\left[C_{2 t}=1 \mid C_{1 t}=1\right] P\left[C_{1 t}=1\right] \\
& +P\left[D_{t} \mid C_{1 t}=1, C_{2 t}=0\right] P\left[C_{2 t}=0 \mid C_{1 t}=1\right] P\left[C_{1 t}=1\right] \\
& P\left[D_{t} \mid C_{2 t}=1\right] P\left[C_{2 t}=1\right]=P\left[D_{t} \mid C_{1 t}=1, C_{2 t}=1\right] P\left[C_{1 t}=1 \mid C_{2 t}=1\right] P\left[C_{2 t}=1\right] \\
& +P\left[D_{t} \mid C_{1 t}=0, C_{2 t}=1\right] P\left[C_{1 t}=0 \mid C_{2 t}=1\right] P\left[C_{2 t}=1\right]
\end{aligned}
$$

Notice that the first terms of both decompositions in (A-5) are identical and represent the contribution of people who have both chronic conditions to the prevalence of disability. For this $\mathrm{K}=2$ case, note that $E_{t}=P\left[D_{t} \mid C_{1 t}=1\right] P\left[C_{1 t}=1\right]+P\left[D_{t} \mid C_{2 t}=1\right] P\left[C_{2 t}=1\right]$.

Thus,

$$
\begin{aligned}
E_{t}= & P\left[D_{t} \mid C_{1 t}=1, C_{2 t}=0\right] P\left[C_{1 t}=1, C_{2 t}=0\right]+ \\
& P\left[D_{t} \mid C_{1 t}=0, C_{2 t}=1\right] P\left[C_{1 t}=0, C_{2 t}=1\right]+ \\
& 2 P\left[D_{t} \mid C_{1 t}=1, C_{2 t}=1\right] P\left[C_{1 t}=1, C_{2 t}=1\right]
\end{aligned}
$$

On the other hand, the true probability of disability attributable to the two conditions, $P\left[D_{t} \mid C_{1 t}+C_{2 t} \geq 1\right]$, can be decomposed as follows:

$$
\begin{gathered}
P\left[D_{t} \mid C_{1 t}+C_{2 t} \geq 1\right]=P\left[D_{t} \mid C_{1 t}=1, C_{2 t}=0\right] P\left[C_{1 t}=1, C_{2 t}=0\right]+ \\
P\left[D_{t} \mid C_{1 t}=0, C_{2 t}=1\right] P\left[C_{1 t}=0, C_{2 t}=1\right]+ \\
P\left[D_{t} \mid C_{1 t}=1, C_{2 t}=1\right] P\left[C_{1 t}=1, C_{2 t}=1\right]
\end{gathered}
$$

Comparing (A-6) and (A-7), it is evident that $E_{t}$ overestimates the portion of disability attributable to chronic conditions by the joint prevalence term, $P\left[D_{t} \mid C_{1 t}=1, C_{2 t}=1\right] P\left[C_{1 t}=1, C_{2 t}=1\right]$. That is, by taking each of the observed chronic conditions singly, $E_{t}$ produces an upper bound on how much disability prevalence can be explained by chronic health conditions. It is easy to generalize this argument to more than two conditions, though the proof requires the introduction of some cumbersome notation. The principle is the same though: $E_{t}$ overcounts relative to $P\left[D_{t} \mid \sum_{k=1}^{K} C_{k t} \geq 1\right]$ because it includes too many joint prevalence terms. Thus, in equation (A-4), $\Delta E_{t}$ measures how an upper bound (to the contribution of observed trends in chronic conditions to disability) changes over time.

While it would be attractive to use equation (A-7) to evaluate exactly how the trends in chronic health explain trends in disability, for practical reasons it is not possible to do so. In particular, to implement our strategy using equation (A-7) would require large 
amounts of data on people with every conceivable set of multiple conditions. In practice, this is impossible since there are many combinations of conditions that are rare in the population. Thus, our strategy is to focus on the most common chronic conditions in the elderly population, and to note our double counting as a limitation of our analysis. 